Revue de l'Institut des langues et cultures

d'Europe, Amérique, Afrique, Asie et Australie

18 | 2013

Les frontières dans le monde hispanique

\title{
Une frontière fluctuante : la diplomatie espagnole et le versatile équilibre des pouvoirs dans les Caraibes entre 1821 et 1868
}

An Unstable Border: Spanish Diplomacy and the Fragile Balance of Power in the Caribbean between 1821 and 1868

\section{Agustín Sánchez Andrés}

Traducteur : Laurie Anne Laget

\section{(2) OpenEdition}

\section{Journals}

Édition électronique

URL : http://journals.openedition.org/ilcea/2060

DOI : 10.4000/ilcea.2060

ISSN : 2101-0609

Éditeur

UGA Éditions/Université Grenoble Alpes

Édition imprimée

ISBN : 978-2-84310-251-6

ISSN : 1639-6073

Référence électronique

Agustín Sánchez Andrés, « Une frontière fluctuante : la diplomatie espagnole et le versatile équilibre des pouvoirs dans les Caraïbes entre 1821 et 1868 », ILCEA [En ligne], 18 | 2013, mis en ligne le 11

juillet 2013, consulté le 19 avril 2019. URL : http://journals.openedition.org/ilcea/2060 ; DOI : 10.4000/ ilcea.2060

Ce document a été généré automatiquement le 19 avril 2019

(c) ILCEA 


\title{
Une frontière fluctuante : la diplomatie espagnole et le versatile équilibre des pouvoirs dans les Caraibes entre 1821 et 1868
}

\author{
An Unstable Border: Spanish Diplomacy and the Fragile Balance of Power in the \\ Caribbean between 1821 and 1868
}

Agustín Sánchez Andrés

Traduction : Laurie Anne Laget

\section{La chute de l'empire espagnol et la nouvelle donne régionale (1821-1847)}

1 La dissolution de l'empire colonial espagnol au cours des premières décennies du XIX siècle marque une nouvelle étape dans la longue série de conflits entre les puissances atlantiques autour du contrôle de la région des Caraibes et du golfe du Mexique. Le fait que l'Espagne devienne une puissance de second rang et le déclin de l'influence française suite à l'indépendance d'Haïti introduisent une rupture dans le délicat équilibre des pouvoirs établi dans cette région au cours du siècle précédent. La nouvelle donne régionale doit compter avec l'apparition des nouvelles nations latino-américaines nées de la fragmentation de l'empire espagnol et dont l'instabilité favorise la montée de l'influence britannique - tout particulièrement en Amérique centrale - ainsi qu'avec une première percée économique et politique des États-Unis. Le problème de l'indéfinition des frontières de ces nouveaux États, aussi bien entre eux (Mexique-Guatemala, République dominicaine-Haïti) qu'avec les États-Unis (pour le Mexique) ou l'empire britannique (pour le Mexique, le Guatemala, le Honduras ou le Nicaragua), conditionne la volatilité des frontières régionales durant le deuxième tiers du XIX ${ }^{\mathrm{e}}$ siècle et fait croître une tendance au conflit déjà marquée dans cette région. À leur tour, le maintien de la domination 
espagnole sur Cuba et Porto Rico et la présence, presque résiduelle, de la France, des Pays-Bas et du Danemark contribuent à augmenter davantage la complexité de la situation des Caraïbes au cours des premières décennies du XIX ${ }^{\mathrm{e}}$ siècle.

Le réajustement de l'équilibre des pouvoirs qu'entraine le processus d'émancipation de l'Amérique continentale fait des Caraïbes le siège d'un singulier affrontement géopolitique entre les différentes puissances qui ont des intérêts en jeu dans cette région. Le refus de Ferdinand VII de reconnaître l'indépendance de ses anciennes colonies vient accroître encore l'instabilité régionale au cours des années 1820 . La menace que représente l'ancienne métropole envers l'indépendance des nouveaux États - affaiblis par une décennie de conflits internes - est propice aux phénomènes de militarisation qui avaient vu le jour dans ces sociétés à la faveur des guerres d'indépendance, tout en obligeant les nouveaux gouvernements à s'endetter à l'extérieur. En retour, l'attitude de l'ancienne métropole conduit le Mexique et la Grande Colombie à conclure une alliance contre l'Espagne. L'accord signé par ces deux républiques, en août 1825, vise à expulser les Espagnols de San Juan de Ulúa, leur dernière possession sur le continent, et à inquiéter la présence espagnole à Cuba ${ }^{1}$. Cette situation amène l'Angleterre à offrir sa médiation, en proposant à l'Espagne de lui garantir le contrôle de Cuba, dès lors qu'elle reconnaît l'indépendance des nouvelles nations hispano-américaines ${ }^{2}$. Le refus espagnol est suivi par l'envoi au Mexique, en 1829, d'une expédition de reconquête ${ }^{3}$. L'échec de cette expédition, dirigée par le militaire Isidro Barradas, incite le gouvernement mexicain à renouer ses liens avec la Grande Colombie pour préparer une invasion conjointe de Cuba, tout en dépêchant un agent mexicain à Haïti dans le but de promouvoir une révolte des esclaves dans les Antilles espagnoles ${ }^{4}$.

3 L'inquiétude de la Grande-Bretagne et des États-Unis face à toute mise en cause du statu quo dans les Caraïbes oblige toutefois le Mexique à renoncer à ses projets de déstabilisation de la présence espagnole à Cuba. En outre, la dissolution de la Grande Colombie, peu de temps après, fait disparaitre toute possibilité d'action conjointe des républiques hispano-américaines à l'encontre des colonies espagnoles dans les Caraïbes. Par ailleurs, le gouvernement mexicain ne parvient pas à négocier son renoncement à une intervention à Cuba contre une garantie anglo-américaine qu'il ne se reproduira plus de nouvelle agression espagnole. En revanche, l'avènement d'une crise de succession en Espagne, en 1830, permet de dissiper définitivement le danger d'une nouvelle expédition de reconquête lancée par l'ancienne métropole 5 .

4 Confronté à de croissantes tensions internes, le monarque espagnol, tout en maintenant son refus à reconnaître l'indépendance de ses anciennes colonies, cesse de représenter un réel danger pour ces dernières. Ferdinand VII, qui est désormais conscient qu'il ne pourra récupérer ses anciens territoires, tente même d'exhumer le Plan d'Iguala. À cette fin, il consulte officieusement le gouvernement mexicain en janvier 1832 sur la possibilité d'asseoir son frère, Charles de Bourbon, sur un hypothétique trône mexicain. Cette utopique proposition répond à un double enjeu : celui de résoudre la crise de succession qu'a fait naître, en Espagne, la naissance d'Isabelle et celui de trouver un allié parmi les anciennes colonies espagnoles ${ }^{6}$.

5 Le ferme refus du Mexique rend impossible tout accord avec les nouvelles républiques hispano-américaines jusqu'à la mort de Ferdinand VII. Alors seulement, la nouvelle stratégie qu'adopte le régime libéral espagnol à ses débuts relance les relations avec les républiques dans le but d'étendre sur elles l'influence espagnole, tout en consolidant la 
position de l'Espagne dans les Antilles, face à la croissante menace que représente la politique expansionniste des États-Unis.

6 Jusqu'à l'expansion territoriale qui sera la conséquence de l'annexion de Texas et de la guerre subséquente américano-mexicaine, la politique des États-Unis dans les Caraïbes visait à maintenir le statu quo. $\mathrm{Ce}$, pour trois raisons. La première est la crainte que toute altération du contrôle exercé par l'Espagne sur Cuba n'entraîne l'abolition de l'esclavage sur l'île, ce qui aurait des répercussions sur la stabilité de la société esclavagiste dans le Sud des États-Unis. La deuxième est l'importance croissante des relations commerciales avec les Grandes Antilles, qui représentent, vers 1840, $50 \%$ des exportations latinoaméricaines vers les États-Unis et $43 \%$ des exportations nord-américaines vers le continent ${ }^{7}$. Enfin, la troisième est l'impuissance de la force navale nord-américaine à défier la Royal Navy dans les Caraïbes. Dans un tel contexte, la politique nord-américaine vise essentiellement à empêcher l'annexion de Cuba par la Grande-Bretagne ou la France et à faire en sorte que les pressions du gouvernement anglais sur les autorités espagnoles n'aboutissent pas au décret de l'abolition de l'esclavage - ce qui aurait des conséquences tout aussi désastreuses. L'implication de fonctionnaires consulaires britanniques, comme David Turnbull ou Francis Ross Cocking, dans la tentative d'organisation, à La Havane, d'un soulèvement d'esclaves, en 1841, redouble les craintes de Washington et assure aux autorités espagnoles la collaboration des consuls nord-américains pour faire face aux pressions britanniques sur Cuba jusqu'à la fin de cette décennie ${ }^{8}$.

7 La politique britannique sur la traite d'esclaves et son abolition est alors dictée à la fois par des considérations humanitaires et par le désir d'éliminer l'avantage dont bénéficient les planteurs cubains par rapport aux producteurs de sucre des Antilles britanniques. Certaines considérations géopolitiques entrent également en jeu, puisque la diplomatie britannique considère que l'abolition de l'esclavage à Cuba ou dans la jeune République du Texas mettrait un frein à l'expansion des États-Unis sur les Caraïbes et le golfe du Mexique. La dissolution de l'empire colonial espagnol ayant pour conséquence, aux yeux des diplomates britanniques, que l'expansionnisme nord-américain constitue désormais un obstacle au maintien de leur influence politique et sociale dans les Caraïbes, ceux-ci soutiennent le maintien de la souveraineté espagnole à Cuba et à Porto Rico, tout en faisant pression sur le gouvernement espagnol pour que celui-ci mette un terme au commerce des esclaves ${ }^{9}$. C'est dans ce but que George Canning tente de promouvoir, en 1825, un traité multilatéral garantissant la domination espagnole sur Cuba, alors que l'île paraissait particulièrement menacée ${ }^{10}$.

8 Les intérêts britanniques s'opposent à ceux des États-Unis non seulement à Cuba mais aussi en Amérique centrale, où l'expansion des frontières de la colonie anglaise de Belize et la création d'un protectorat britannique de facto à La Mosquitia ont nourri la défiance des États-Unis envers la politique britannique dans une région cruciale pour la construction du futur canal interocéanique ${ }^{11}$.

9 La principale bénéficiaire de l'équilibre des pouvoirs entre les États-Unis et la GrandeBretagne est l'Espagne. L'ancienne puissance coloniale est parvenue à conserver Cuba et Porto Rico, notamment grâce à la crainte des grands propriétaires terriens créoles de se trouver confrontés à une révolte d'esclaves. Au cours du deuxième quart du XIX ${ }^{\mathrm{e}}$ siècle, la politique coloniale espagnole est déterminée par la fragilité de la métropole elle-même, encore sous les effets de l'occupation française de 1808 à 1814, ainsi que ceux de la perte de l'empire colonial. En outre, la mort de Ferdinand VII, en 1834, ouvre un long conflit entre les libéraux et les partisans de l'Ancien Régime, qui se traduit sous la forme de deux 
guerres civiles, en 1834-1839 puis en 1846-1849, et vient renforcer la dépendance financière de l'Espagne vis-à-vis de la Grande-Bretagne et de la France. En ce sens, les premiers temps du processus de création d'un État-nation libéral dans la Péninsule sont marqués par une grande instabilité interne. À son tour, cette fragilité de l'État espagnol pendant le deuxième quart $\mathrm{du} \mathrm{XIX}^{\mathrm{e}}$ siècle entraîne l'autonomie des autorités de Cuba et de Porto Rico vis-à-vis de la métropole et fait du maintien de l'esclavage la base d'un nouveau pacte colonial avec les planteurs créoles qui assure le contrôle de l'Espagne sur ces deux îles ${ }^{12}$.

Dans un tel contexte, la politique espagnole dans les Caraibes au cours des années 1830-1840 vise essentiellement à tirer parti de l'intérêt qu'ont alors les États-Unis, la Grande-Bretagne et la France à ce qu'aucune des deux autres puissances n'annexe Cuba. Le maintien de cet équilibre des pouvoirs, tout autant que de la précaire situation de l'Espagne à Cuba, exigent également celui de l'esclavage sur l'île. Cela implique un double jeu avec la Grande-Bretagne, qui consiste à céder en apparence aux pressions britanniques en interdisant formellement la traite des esclaves, sans que cette interdiction de la part de la métropole ne soit réellement mise en application, étant donné la connivence des autorités cubaines avec le trafic négrier, devenu un enjeu en raison de l'essor vertigineux de l'économie sucrière sur l'île ${ }^{13}$.

11 En parallèle, les différents gouvernements espagnols de l'époque, progressistes ou modérés, relancent les relations avec les jeunes républiques des Caraïbes dans le but de renforcer le contrôle de l'Espagne sur Cuba et sur Porto Rico. C'est la raison pour laquelle ils reconnaissent l'indépendance du Mexique, en 1836, et celle du Venezuela, en 1845, ainsi que l'attestent les dispositions secrètes obligeant les nouvelles nations à respecter la souveraineté espagnole dans les Antilles. Par ailleurs, les autorités espagnoles ne tardent pas à vouloir étendre leur influence sur les nouvelles républiques dans le but de s'assurer des alliés locaux et de se prémunir contre les autres puissances impliquées dans cette région. C'est dans ce cadre qu'il faut analyser l'ambivalence de la politique mise en place par l'Espagne en République dominicaine de 1844 à 1855 et, surtout, la conspiration monarchique (qui finalement échouera) lancée par le gouvernement espagnol visant à asseoir sur le trône du Mexique, en 1846, un infant d'Espagne ${ }^{14}$.

\section{La stratégie espagnole en réponse à l'expansionnisme nord-américain dans les Caraïbes (1846-1860)}

12 La situation change de façon radicale à partir du moment où les États-Unis révisent leur politique à l'égard des Caraibes, à la suite de la guerre américano-mexicaine et de l'annexion de la moitié de ce pays conformément au traité de Guadaloupe Hidalgo de 1848. Un virulent mouvement expansionniste, fondé sur la notion de " destin manifeste ", se développe alors, en particulier parmi la classe politique et dans la société du Sud des États-Unis. Cela amène les différentes administrations qui se succèdent, de James K. Polk à James Buchanan, à renoncer à une politique centrée sur le maintien du statu quo dans les Caraibes pour tenter, au contraire, d'établir l'hégémonie des États-Unis dans cette région. L'acquisition de Cuba et, dans une moindre mesure, celle d'autres territoires comme la République dominicaine ou les Îles vierges, devient l'objectif principal de la nouvelle politique des États-Unis dans la région ${ }^{15}$. 
13 En ce qui concerne Cuba, la politique nord-américaine consiste à faire pression sur le gouvernement espagnol pour que celui-ci vende l'île aux États-Unis. Le développement d'un mouvement annexionniste au sein de l'oligarchie antillaise, qui redoute que le gouvernement espagnol ne cède aux pressions de la Grande-Bretagne et décrète l'abolition de l'esclavage, vient appuyer cette stratégie. Le soutien que ces annexionnistes cubains trouvent au Sud des États-Unis crée des tensions dans les relations avec l'Espagne, de 1848 à $1855^{16}$.

14 La première proposition d'achat est lancée, en 1848, par l'administration de James K. Polk. Le refus de l'Espagne entraîne l'organisation, depuis les États-Unis, de trois expéditions contre Cuba, lancées entre 1849 et 1851 dans le but explicite d'annexer l'île.

15 Le gouvernement espagnol y répond en renforçant son dispositif militaire dans les Caraibes et fait front aux expéditions annexionnistes organisées par Narciso López. L'exécution de plusieurs dizaines d'envoyés nord-américains manque alors de provoquer un conflit entre les deux pays. Ce qui conduit la diplomatie espagnole à chercher à obtenir des garanties internationales sur ses colonies antillaises. Le gouvernement britannique, qui vient alors d'obtenir de Washington un répit en Amérique centrale grâce à la signature du traité Clayton-Bulwer, en 1850, semble favorable à un accord international pour éviter le danger d'une hypothétique annexion de Cuba par les ÉtatsUnis. Néanmoins, les négociations échouent en raison du refus de l'Espagne à abolir l'esclavage dans les Antilles - condition imposée par Palmerston - et du commencement, en 1854, de la guerre de Crimée, qui détourne l'attention de l'Angleterre et de la France de la question caribéenne ${ }^{17}$.

16 Tout cela laisse une plus grande marge de manœuvre à la mise en place de la politique nord-américaine, de plus en plus agressive, dans les Caraïbes. De 1853 à 1854, l'administration de Franklin Pierce conduit l'Espagne et les États-Unis au bord du conflit ouvert. Le gouvernement espagnol refuse chaque nouvelle proposition d'achat nordaméricaine, malgré les menaces d'intervention sur l'île qui les accompagnent, et pour éviter toute nouvelle expédition indépendantiste, il établit un blocus naval autour de Cuba, qui ne manque pas d'affecter le trafic commercial entre l'île et les États-Unis, obligeant finalement Pierce à renoncer provisoirement à ses projets d'achat pour ouvrir des négociations en vue de signer un traité commercial ${ }^{18}$.

17 En parallèle, l'Espagne tente de faire face à la pression croissante des États-Unis sur Cuba et Porto Rico en étendant son aire d'influence sur d'autres pays de la région, comme le Mexique et la République dominicaine, afin de contrecarrer l'expansionnisme nordaméricain sur ses colonies antillaises ${ }^{19}$. La stratégie espagnole est toutefois mise en difficulté par le fait que l'Espagne, à la différence de son rival nord-américain, a rarement eu l'occasion de s'impliquer directement dans ces autres conflits où s'arbitre le combat entre les deux puissances dans les Caraïbes. Consciente de cette faiblesse, l'Espagne poursuit sa politique centrée sur l'enjeu que représente, pour la France et l'Angleterre, le maintien du délicat équilibre des pouvoirs existant dans la région, qu'un conflit entre Madrid et Washington viendrait mettre en péril.

18 L'Espagne se trouve ainsi freinée dans son ambition d'étendre son influence sur le Mexique, où l'ancienne métropole disposait pourtant d'importants soutiens au sein du Parti conservateur dirigé par Lucás Alamán qui, en $1846^{20}$, puis en 1853 et $1854^{21}$ tente d'établir une monarchie avec à sa tête l'infant d'Espagne. La guerre contre les États-Unis et l'instabilité politique mexicaine viendront à bout de ces projets favorables aux intérêts 
espagnols, visant à établir un régime monarchique au Mexique. C'est ainsi que Madrid doit se résigner à constater l'effet des pressions diplomatiques exercées par Washington, en 1853, et de l'implication directe de militaires nord-américains dans la guerre de Réforme, en 1860 : l'Espagne voit échouer ses projets interventionnistes tandis que le Mexique passe sous la sphère d'influence des États-Unis. Plus alarmant encore, un tel contexte ouvre la voie à de nouvelles acquisitions de territoire mexicain par les ÉtatsUnis, comme l'attestent l'achat de Mesilla, en décembre 1853, et la signature du traité McLane-Ocampo, qui hypothèque, en décembre 1859, d'importantes parcelles de la souveraineté mexicaine et octroie aux États-Unis un droit de transit sur l'isthme de Tehuantepec.

19 La situation est plus ou moins similaire en République dominicaine où, après l'indépendance d'Haitti, en 1844, une partie de l'élite conserve encore de forts liens identitaires avec l'ancienne métropole ${ }^{22}$. La diplomatie espagnole met à profit ces sentiments en signant, en 1855, un traité qui, s'il avait été appliqué dans son intégralité, aurait établi un protectorat de fait sur la république caribéenne ${ }^{23}$. De 1856 à 1858 , l'Espagne intervient en faveur de l'un ou l'autre des deux grands caudillos dominicains rivaux, Pedro Santana et Buenaventura Báez, pour finalement s'apercevoir que, dans un cas comme dans l'autre, le parti pro espagnol est renversé par les partisans de l'annexion du pays par la puissance américaine ${ }^{24}$.

\section{L'infructueuse réaction européenne et le réajustement de l'équilibre des pouvoirs (1861-1868)}

20 Le début de la guerre de Sécession, en avril 1861, vient interrompre la progression de l'influence nord-américaine sur les Caraïbes et rend possible une contre-offensive européenne. La France et l'Espagne, tout particulièrement, mettent à profit cette nouvelle donne pour tenter de réaffirmer leur présence dans la région au cours de la première moitié des années 1860 .

21 La guerre civile américaine et le retrait momentané des États-Unis de l'échiquier international permettent la mise en application du projet de Napoléon III pour étendre son influence sur le continent américain, faisant du Mexique un État satellite susceptible de servir de barrière à l'expansionnisme nord-américain dans la région, tout en jetant les bases de l'implantation de régimes monarchiques sous influence française dans d'autres pays du continent, comme l'Équateur ${ }^{25}$. La constitution d'un État-satellite au Mexique pouvait également permettre à la France de construire et de contrôler un canal interocéanique entre le Pacifique et l'Atlantique ${ }^{26}$. Ce vaste et utopique projet s'inscrit dans le cadre des efforts de l'empereur français, tout au long de son règne, pour parvenir à une hégémonie politique et morale sur le dit «monde latin » et assurer à la France le rôle de grande puissance mondiale, en réunissant le peuple français autour du projet impérial ${ }^{27}$.

En Espagne, la crise interne aux États-Unis coïncide avec la permanence au pouvoir de l'Union libérale, le parti fondé par Leopoldo O'Donnell qui, depuis 1858, gouverne l'Espagne. Cette période de stabilité politique, aussi longue qu'inhabituelle, s'accompagne, par ailleurs, d'un cycle d'expansion économique qui perdurera jusqu'en $1866^{28}$. Simultanément, les prétentions annexionnistes sur Cuba se réduisent alors que s'ébauche un rapprochement entre l'élite créole cubaine et les secteurs du libéralisme espagnol, 
apparemment disposés à intégrer les membres de cette élite à la base de consensus du régime d'Isabelle II ${ }^{29}$.

C'est à la faveur de ce contexte qu'o'Donnell se lance dans une intense activité diplomatique et militaire sur le continent américain, dans le but de renforcer la présence espagnole dans les Caraïbes et le golfe du Mexique tout autant que de consolider la popularité de l'Union libérale en Espagne à travers une «diplomatie de prestige ", selon la formule de Durán de la Rua, qui ne se limite pas uniquement à l'Amérique, mais englobe également le Maroc et le Sud-Est de l'Asie ${ }^{30}$.

En 1861-1862, l'Espagne participe de cette façon, aux côtés de la France et de l'Angleterre, à l'intervention tripartite sur le Mexique, suite au non-paiement de la dette externe par le gouvernement de Benito Juárez. Le gouvernement espagnol, tout comme le gouvernement britannique, retireront leurs troupes dès que le projet de Napoléon III de faire du Mexique un État satellite aura été découvert. Cela n'empêchera pas Madrid de reconnaître peu après le régime de Maximilien d'Autriche, en qui elle voit un solide allié face à l'expansionnisme nord-américain ${ }^{31}$. Cette même année, le gouvernement espagnol se lance dans la ré-annexion de la République dominicaine, à la requête d'un important secteur de l'élite dominicaine ayant à sa tête Pedro Santana, le président du pays luimême ${ }^{32}$. Le gouvernement espagnol avait auparavant obtenu l'approbation de Napoléon III, soucieux de recouvrer le soutien espagnol pour son aventure mexicaine, ainsi que celle de Lord Russell, qui avait obtenu des nouvelles autorités espagnoles la garantie que celles-ci ne rétabliraient pas l'esclavage à Saint-Domingue ${ }^{33}$. L'administration de Lincoln, qui redoute que l'Europe reconnaisse la Confédération, assiste, impuissante, au défi européen lancé à la doctrine Monroe et se contente d'envoyer une note de protestation, dont elle fait parvenir une copie aux gouvernements latino-américains ${ }^{34}$. Trois ans plus tard, un bataillon espagnol prend le pouvoir sur les îles Chinchas et transforme la crise diplomatique avec le Pérou en un conflit généralisé avec les républiques du Chili, du Pérou, de l'Équateur et de la Bolivie. Dans le cadre de ce conflit, la flotte espagnole bloque durant presque un an les principaux ports du Sud du Pacifique et bombarde Valparaíso et El Callao ${ }^{35}$.

Cette offensive politico-diplomatique espagnole en Amérique aura, finalement, pour effet d'augmenter la méfiance des jeunes républiques latino-américaines à l'égard de l'ancienne métropole ${ }^{36}$. La fin de la guerre de Sécession et l'usure progressive du régime d'Isabelle II vont causer un nouveau repli de l'influence espagnole sur l'Amérique latine, qui se fera tout particulièrement sentir dans les Caraïbes et le golfe du Mexique. Dans ce contexte, l'Espagne se voit obligée, en 1865, à faire évacuer la République dominicaine, face à l'impossibilité de maîtriser le soulèvement ayant commencé en 1863 et qui s'était progressivement nourri des attentes déçues par le retour éphémère de la souveraineté espagnole $^{37}$. La chute de l'empire de Maximilien d'Autriche, deux ans plus tard, et le regain des projets annexionnistes de Washington sur la République dominicaine et sur Haïti viennent menacer le fragile équilibre régional dont dépend la présence espagnole à Cuba.

Le dernier cabinet unioniste espagnol tente de repousser ce danger en mettant un terme aux ambitions séparatistes de l'élite antillaise par la réforme des institutions coloniales de Cuba et de Porto Rico ${ }^{38}$. Mais ce projet ne parviendra pas à son terme. La chute de Leopoldo O'Donnell, au cours de l'été 1866, transfert le pouvoir aux secteurs les plus intransigeants du modérantisme, alors dirigés par Ramón María de Narváez, et qui, en 
s'opposant à toute remise en cause du statu quo colonial, rendent plus délicate encore la position de l'Espagne dans les Antilles.

Durant les premiers mois de l'année 1868, la diplomatie espagnole doit faire face à une situation de plus en plus problématique dans les Caraïbes. Tout d'abord, en raison des insistants projets de l'administration américaine visant à établir une base navale en République dominicaine ou à Haïti à travers la location ou l'achat de diverses enclaves stratégiques dans l'une ou l'autre des républiques. Par ailleurs, aux inquiétantes négociations menées par les États-Unis s'ajoutent celles des diplomates des républiques d'Amérique du Sud limitrophes des Caraïbes, avec lesquels l'Espagne est déclarée en guerre depuis $1865^{39}$.

Il s'agit, dans un cas comme dans l'autre, de menaces directes à la sécurité des colonies antillaise de l'Espagne. D'une part, la mise en place d'une station navale permanente à Saint-Domingue assurerait aux États-Unis le contrôle d'une île stratégique, située entre Cuba et Porto Rico, dont la situation géographique permettrait, en cas de conflit, d'interrompre les communications entre les colonies espagnoles et la Péninsule. D'autre part, les gouvernements du Pérou et du Chili cherchaient à convaincre le Venezuela et la Colombie de former une alliance pour expulser l'Espagne d'Amérique, tout en subventionnant l'exil cubain afin de déstabiliser les colonies espagnoles des Caraïbes. Ces négociations conduiront à la signature du traité secret d'alliance entre la Colombie et le Pérou, en $1866^{40}$.

Toutefois, la principale source d'inquiétude pour les autorités espagnoles reste celle des projets annexionnistes de Washington à Saint-Domingue. À la fin de la guerre de Sécession, les autorités nord-américaines décident de rattraper au plus vite le terrain perdu dans les Caraïbes. Peu après le retrait espagnol, le secrétaire d'État, William $\mathrm{H}$. Seward, relance les projets d'achat de la baie de Samaná et envoie à cette fin son fils, Frederick Seward, sur l'île ${ }^{41}$. L'inquiétude de la diplomatie espagnole est tout à fait fondée, puisque, depuis 1866, le régime dominicain de José María Cabral a ouvert des négociations secrètes avec les États-Unis pour le bail de la baie de Samaná. Avec le retour au pouvoir de Báez, en février 1868, le rythme des négociations s'accélère ${ }^{42}$. Par ailleurs, la diplomatie espagnole a également à redouter les tractations menées par Washington auprès du président haïtien Sylvain Salnave pour l'achat du Môle Saint-Nicolas ${ }^{43}$.

30 Cette configuration oblige le gouvernement espagnol à chercher l'appui des autres puissances ayant des intérêts en jeu dans la région. À l'automne 1868, Madrid donne l'ordre à ses représentants à Londres d'évaluer la position du gouvernement britannique concernant les intentions des États-Unis à Saint-Domingue, probablement dans le but de promouvoir une action conjointe pour faire échouer les projets nord-américains dans l'ancienne Hispaniola. En réalité, le temps d'une intervention collective européenne dans les Caraïbes est révolu et le gouvernement britannique, tout en partageant les inquiétudes de Madrid, fait comprendre aux autorités espagnoles qu'il n'est pas disposé à adopter la moindre mesure susceptible de provoquer un conflit avec les États-Unis ${ }^{44}$.

31 L'échec de ces pourparlers conduit l'Espagne à tenter de renforcer sa position dans les Caraïbes en ébauchant un timide rapprochement avec les différentes républiques de cette région. Le gouverneur de Cuba, Francisco Lersundi, fait le premier pas pour rétablir les relations avec le Mexique - interrompues depuis la chute de l'empire de Maximilien d'Autriche - lorsqu'en 1868 il expulse de l'île Antonio López de Santa Anna pour avoir conspiré contre le gouvernement restauré de Juárez ${ }^{45}$. Lersundi se lance, par ailleurs, dans des négociations pour rendre au Mexique les bâtiments de guerre impériaux qui 
s'étaient réfugiés dans les ports de Cuba après la chute du régime de Maximilien d'Autriche ${ }^{46}$. Le ministre d'État, de son côté, commence à étudier les possibles solutions aux contentieux hispano-dominicains qui, depuis la ré-annexion de ce territoire par l'Espagne, empêchaient toute relation directe entre les deux pays. Pour cela, il se montre favorable à la médiation entreprise par les États-Unis dans le but de mettre fin aux conflits avec les républiques du Pacifique ${ }^{47}$.

Le changement de cap de la politique extérieure de l'Espagne dans les Caraïbes ne parviendra cependant pas à son terme, en raison de la chute du régime d'Isabelle II au lendemain de la révolution libérale, en septembre 1868. Un mois plus tard, deux révolutions indépendantistes éclatent, à Porto Rico et à Cuba. Les autorités coloniales ne parviendront à étouffer que la première. C'est alors que s'ouvre, en Espagne, une longue période d'instabilité politique, au cours du Sexenio Revolucionario. Finalement, l'incapacité de cette puissance à mettre un terme à la rébellion cubaine, entre 1868 et 1881, entraînera la rupture définitive de l'équilibre des pouvoirs dans les Caraïbes.

\section{Conclusions}

La dissolution de l'empire colonial espagnol est à l'origine d'un réajustement du fragile équilibre des pouvoirs dans les Caraïbes et d'une recrudescence des tensions géopolitiques entre l'Espagne, les États-Unis, l'Angleterre et la France pour obtenir le contrôle de cette région stratégique. La politique espagnole dans les Caraïbes doit s'adapter à une nouvelle donne régionale marquée par la présence espagnole restreinte à Cuba et à Porto Rico, l'apparition de nouveaux acteurs représentés par les nations hispano-américaines, le déclin de l'influence française dans la région au lendemain de l'indépendance d'Haïti et les débuts de la pénétration politique et économique des ÉtatsUnis. Le refus de l'Espagne à reconnaître l'indépendance de ses anciennes colonies rend la situation plus complexe encore jusqu'à la mort de Ferdinand VII, en décembre 1834. Dans ce contexte, le maintien de la domination espagnole sur Cuba et Porto Rico oblige la diplomatie espagnole à mettre en pratique diverses stratégies de nature défensive: depuis chercher des garanties auprès de la France et de la Grande-Bretagne jusqu'à étendre son influence sur les nouvelles nations hispano-américaines dans le but de trouver des alliés avec qui faire face à la croissante pression des États-Unis sur Cuba.

Toutes ces stratégies échoueront face à l'agressive politique entreprise par les États-Unis dans la région, dont l'objectif est l'annexion d'une partie du Mexique et de la République dominicaine, ainsi que de la totalité de la colonie espagnole de Cuba. L'incapacité des puissances européennes à contrecarrer l'expansionnisme grandissant des États-Unis dans les Caraïbes, à laquelle vient s'ajouter l'extrême fragilité de la situation espagnole à Cuba à partir de 1868, conduit irréversiblement à la rupture de l'équilibre des pouvoirs qui, depuis le début $d u \mathrm{xIX}^{\mathrm{e}}$ siècle, existait dans cette région. C'est ainsi que s'établira l'influence nord-américaine sur les Caraïbes, après la brève parenthèse de la guerre de Sécession qui avait donné un répit à la présence européenne. 


\section{BIBLIOGRAPHIE}

\section{Archives consultées}

AMAE : Archivo del Ministerio de Asuntos Exteriores, Madrid

\section{Bibliographie}

AMORES Juan B., Cuba y España, 1868-1898. El final de un sueño, Pampelune, Universidad de Navarra, 1998.

BARTLETT Christopher John, Great Britain and Sea Power, 1815-1853, Oxford, Clarendon Press, 1967. BELINKI Alexander, La intervención francesa en México, 1861-1867, Mexique, Quinto Sol, 1986.

BOURNE K., Britain and the Balance of Power in North America, 1815-1908, Berkeley, University of California Press, 1967.

CASTEL Jorge, Anexión y abandono de Santo Domingo (1810-1865), Madrid, Cuadernos de Historia de las Relaciones Internacionales y Política Exterior de España, 1954.

CORTADA James W., Two Nations Over Time. Spain and the United States, 1776-1977, Wesport-Londres, Greenwood Press, 1978a.

-, « España y Estados Unidos ante la cuestión mexicana, 1855-1868 », Historia Mexicana (México), vol. XXVII, nº 3, 1978b, p. 383-410.

-, « The United States ", dans J. W. cortada (éd.), Spain in the Nineteenth-Century World. Essays on Spanish Diplomacy, 1789-1898, Londres, Greenwood Press, 1994.

DELGADO LARIOS Almudena, « Justicia y relaciones internacionales: las relaciones hispanomexicanas (1844-1863) », Anuario de Estudios Americanos, vol. 66, n 1, 2009, p. 47-78.

DURÁN Nelson, La Unión Liberal y la modernización de la España isabelina. Una convivencia frustrada, 1854-1868, Madrid, Akal, 1979.

FOWLER Will, Santa Anna of Mexico, Lincoln, University of Nebraska Press, 2007.

FRADERA Josep María, Colonias para después de un imperio, Barcelone, Bellatera, 2005.

GONZÁLEZ Moisés, Los extranjeros en México y los mexicanos en el extranjero, 1821-1970, Mexique, El Colegio de México, 1993.

HAUch Charles Christian, La República Dominicana y sus relaciones exteriores, 1844-1882, Santo Domingo, Sociedad Dominicana de Bibliófilos, 1996.

INAREJOS MUÑOZ Juan Antonio, Intervenciones coloniales y nacionalismo español. La política exterior de la Unión Liberal, Madrid, Sílex, 2010.

LANGLEY Lester D., Struggle for the American Metiterranean. United States-European Rivalry in the GulfCaribbean, 1776-1904, Athens, The University of Georgia Press, 1976.

LECAILLON Jean-Francois, Napoléon III et le Mexique. Les illusions d'un grand dessein, Paris,

L'Harmattan, 1994. 
LÓPEZ OCÓN CABRERA Leoncio, Biografía de La «América». Una crónica hispano-americana del liberalismo democrático español (1857-1886), Madrid, CSIC, 1987.

MARTÍNEZ-FERNÁNDEZ Luis, Torno between Empires. Economy, Society and Patterns of Political Thought in the Hispanic Caribbean, 1840-1878, Athens-Londres, The University of Georgia Press, 1994.

MEYER Jean, Francia y América, Madrid, Mapfre, 1992.

MURRAY David R., Odious Commerce: Britain, Spain and the Abolition of the Cuban Slavery, Cambridge, Cambridge University Press, 1976.

NAVARRO Luis, La Independencia de Cuba, Madrid, Mapfre, 1992.

NÚÑEZ Diómedes, Anexionismo y resistencia. Relaciones dominico-norteamericanas en tiempos de Grant, Báez y Luperón, Saint-Domingue, Alfa y Omega, 1997.

PAQUETTE Robert L., Sugar is Made with Blood: The Conspiracy of La Escalera and the Conflict Between empires over Slavery in Cuba, Middletown, Wesleyan University Press, 1988.

PÉREZ Carlos F., Historia diplomática de Santo Domingo, Saint-Domingue, Universidad Nacional Pedro Enríquez Ureña, 1998.

PI-SUÑER Antonia et SÁNCHEZ Agustín, Una historia de encuentros y desencuentros. México y España en el siglo XIX, Mexique, Secretaría de Relaciones Exteriores, 2001.

RIVAS Raimundo, Historia diplomática de Colombia (1810-1934), Bogotá, Ministerio de Relaciones Exteriores, 1961.

ROBERTSON W. S., Hispanic-American Relations with the United States, New York, Oxford University Press, 1923.

ROBLES Cristobal, Paz en Santo Domingo, 1854-1865. El fracaso de la anexión a España, Madrid, Consejo Superior de Investigaciones Científicas, 1987.

RODRÍGUEZ Emilio, Relaciones dominico-españolas (1844-1859), Ciudad Trujillo, [s. é.], 1955.

ROLDÁN Ornán, Las relaciones entre México y Colombia, 1810-1862, Mexique, Secretaría de Relaciones Exteriores, 1974.

RUBIO Javier, El Gobierno español en busca de una garantía internacional sobre Cuba en vísperas del 98, Madrid, Comisión Española de Historia de las Relaciones Internacionales, 1998.

SÁNCHEZ Agustín, « De la Intervención Tripartita a la caída del Imperio », dans Clara E. Lida (coord.), España y el Imperio de Maximiliano, Mexique, El Colegio de México, 1999.

-, « De la independencia al reconocimiento. Las relaciones hispano-mexicanas entre 1820 y 1836 ", dans Agustín Sánchez et Raul Figueroa (coord.), México y España en el siglo XIX. Diplomacia, relaciones triangulares e imaginarios nacionales, Mexique, Universidad Michoacana de San Nicolás de Hidalgo et Instituto Tecnológico Autónomo de México, 2003.

-, «Colonial Crisis and Spanish Diplomacy in the Caribbean During the Sexenio Revolucionario, 1868-1874 », Bulletin of Latin American Research, vol. 28, n 3, 2009, p. 325-342.

SÁNCHEZ José, La España contemporánea, Madrid, Istmo, 1991.

SANTOVEnIA Emeterio, Armonías y conflictos en torno a Cuba, Mexique, Fondo de Cultura Económica, 1956.

SCHIMDT-NOWARA Christopher, The conquest of history: Spanish colonialism and national histories in the nineteenth century, Pittsburgh, University of Pittsburgh Press, 2006. 
SCHOONOVER Thomas, "Latin America ", dans James W. Cortada (éd.), Spain in the NineteenthCentury World. Essays on Spanish Diplomacy, 1789-1898, Wesport et Londres, Greenwood Press, 1994. SIMS Harlod D., La reconquista de México. La historia de los atentados españoles, Mexique, Fondo de Cultura Económica, 1984.

Sото Miguel, La conspiración monárquica en México, 1845-1848, Mexique, EOSA, 1988.

SUÁREZ Juan, Historia de México y del general Santa Anna. Comprende los acontecimientos que han tenido lugar en la Nación desde el año 1821 hasta 1848, Mexique, Imprenta de Ignacio Cumplido, 1850.

SUMNER Benjamin, Naboth's Vineyard: The Dominican Republic, 1844-1924, New York, Payson \& Clark, 1928.

VAN AKEN Mark J., Pan-Hispanism. Its Origin and Development to 1866, Berkeley-Los Angeles, University of California Press, 1959.

ZAMACoIS Niceto de, Historia de México desde sus tiempos más remotos hasta nuestros días, Barcelone, J. F. Parrés y Cía, 1879.

\section{NOTES}

1. O. Roldán, Las relaciones entre México y Colombia, 1810-1862, 1974, p. 66-67.

2. A. Sánchez, « De la independencia al reconocimiento. Las relaciones hispano-mexicanas entre 1820 y 1836 ", dans A. Sánchez et R. Figueroa (coord.), México y España en el siglo XIX. Diplomacia, relaciones triangulares e imaginarios nacionales, Mexique, Universidad Michoacana de San Nicolás de Hidalgo et Instituto Tecnológico Autónomo de México, 2003, p. 36.

3. À propos de la genèse et du déroulement de l'expédition de de Barradas, voir: J. Suárez, Historia de México y del general Santa Anna, 1850, p. 416-425 ; N. Zamacois, Historia de México desde su independencia hasta nuestros días, 1879, vol. XIX, p. 721-725 et M. González, Los extranjeros en México, 1993, p. 92-97.

4. H. D. Sims, La reconquista de México. La historia de los atentados españoles, Mexique, Fondo de Cultura Económica, 1984, p. 121-162.

5. A. Sánchez, « De la independencia al reconocimiento. Las relaciones hispano-mexicanas entre 1820 y 1836 ", art. cité, p. 39-40.

6. Ibid., p. 42.

7. W. S. Robertson, Hispanic-American Relations with the United States, New York, Oxford University Press, 1923, p. 420.

8. D. R. Murray, Odious Commerce: Britain, Spain and the Abolition of the Cuban Slavery, Cambridge, Cambridge University Press, 1976, p. 169-170.

9. K. Bourne, Britain and the Balance of Power in North America, 1815-1908, Berkeley, University of California Press, 1967, p. 63-69.

10. C. J. Bartlett, Great Britain and Sea Power, 1815-1853, Oxford, Clarendon Press, 1967, p. 69-70.

11. L. D. Langley, Struggle for the American Metiterranean. United States-European Rivalry in the GulfCaribbean, 1776-1904, Athens, The University of Georgia Press, 1976, p. 84-89.

12. L. Martínez-Fernández, Torno between Empires. Economy, Society and Patterns of Political Thought in the Hispanic Caribbean, 1840-1878, Athens-Londres, The University of Georgia Press, 1994, p. 17-18.

13. Voir R. L. Paquette, Sugar is Made with Blood: The Conspiracy of La Escalera and the Conflict Between empires over Slavery in Cuba, Middletown, Wesleyan University Press, 1988.

14. Voir M. Soto, La conspiración monárquica en México, 1845-1848, Mexique, EOSA, 1988. 
15. J. W. Cortada, Two Nations Over Time. Spain and the United States, 1776-1977, Wesport-Londres, Greenwood Press, 1978a, p. 64.

16. L. Martínez-Fernández, Torno between Empires. Economy, Society and Patterns of Political Thought in the Hispanic Caribbean, 1840-1878, ouvr. cité, p. 49-51.

17. J. Rubio, El Gobierno español en busca de una garantía internacional sobre Cuba en vísperas del 98, Madrid, Comisión Española de Historia de las Relaciones Internacionales, 1998, p. 3-8.

18. J. W. Cortada, "The United States ", dans J. W. Cortada (éd.), Spain in the Nineteenth-Century World. Essays on Spanish Diplomacy, 1789-1898, Londres, Greenwood Press, 1994, p. 73.

19. J. W. Cortada, «España y Estados Unidos ante la cuestión mexicana, 1855-1868 », Historia Mexicana (México), vol. XXVII, nº 3, 1978b, p. 387-389.

20. Voir M. Soto, La conspiración monárquica en México, 1845-1848, ouvr. cité.

21. A. Pi-Suñer et A. Sánchez, Una historia de encuentros y desencuentros. México y España en el siglo XIX, Mexique, Secretaría de Relaciones Exteriores, 2001, p. 103-109.

22. E. Rodríguez, Relaciones dominico-españolas (1844-1859), Ciudad Trujillo, [s. é.], 1955, p. 3-5.

23. Le problème venait de l'article 7 de ce traité qui définissait les prérequis nécessaires pour être enregistré comme Espagnol et dont l'interprétation expansive aurait permis à une grande partie de la population de s'enregistrer comme Espagnols. Pour consulter le texte de ce traité, voir J. Castel, Anexión y abandono de Santo Domingo, 1954, p. 41-60.

24. B. Sumner, Naboth's Vineyard: The Dominican Republic, 1844-1924, New York, Payson \& Clark, 1928, p. 192-194 et C. Robles, Paz en Santo Domingo, 1854-1865. El fracaso de la anexión a España, Madrid, Consejo Superior de Investigaciones Científicas, 1987, p. 33-44.

25. J.-F. Lecaillon, Napoléon III et le Mexique. Les illusions d'un grand dessein, Paris, L'Harmattan, 1994, p. 45-47.

26. A. Belinki, La intervención francesa en México, 1861-1867, Mexique, Quinto Sol, 1986, p. 59-61.

27. J. Meyer, Francia y América, Madrid, Mapfre, 1992, p. 210.

28. J. Sánchez, La España contemporánea, Madrid, Istmo, 1991, p. 436-441.

29. L. Navarro, La Independencia de Cuba, Madrid, Mapfre, 1992, p. 251-256.

30. N. Durán, La Unión Liberal y la modernización de la España isabelina. Una convivencia frustrada, 1854-1868, Madrid, Akal, 1979, p. 227-229.

31. A. Sánchez, « De la Intervención Tripartita a la caída del Imperio », art. cité, p. 105-178.

32. C. Robles, Paz en Santo Domingo, 1854-1865. El fracaso de la anexión a España, ouvr. cité, p. 109-213.

33. C. C. Hauch, La República Dominicana y sus relaciones exteriores, 1844-1882, Santo Domingo, Sociedad Dominicana de Bibliófilos, 1996, p. 127-129.

34. L. D. Langley, Struggle for the American Metiterranean. United States-European Rivalry in the GulfCaribbean, 1776-1904, ouvr. cité, p. 142.

35. J. W. Cortada, «España y Estados Unidos ante la cuestión mexicana, 1855-1868 », art. cité, p. 387-425.

36. T. Schoonover, « Latin America », dans James W. Cortada (éd.), Spain in the Nineteenth-Century World. Essays on Spanish Diplomacy, 1789-1898, Wesport et Londres, Greenwood Press, 1994, p. 117.

37. C. F. Pérez, Historia diplomática de Santo Domingo, Saint-Domingue, Universidad Nacional Pedro Enríquez Ureña, 1998, II, p. 13-24.

38. J. B. Amores, Cuba y España, 1868-1898. El final de un sueño, Pampelune, Universidad de Navarra, 1998, p. 52-61.

39. E. Santovenia, Armonías y conflictos en torno a Cuba, Mexique, Fondo de Cultura Económica, 1956, p. 154-190.

40. R. Rivas, Historia diplomática de Colombia (1810-1934), Bogotá, Ministerio de Relaciones Exteriores, 1961, p. 432-434.

41. L. D. Langley, Struggle for the American Metiterranean. United States-European Rivalry in the GulfCaribbean, 1776-1904, ouvr. cité, 1976, p. 142-143. 
42. D. Nuñez, Anexionismo y resistencia. Relaciones dominico-norteamericanas en tiempos de Grant, Báez y Luperón, Saint-Domingue, Alfa y Omega, 1997, p. 166.

43. Voir le courrier daté du 24 juin 1868 de Mariano Álvarez, consul général à Haïti, au ministre d'État, dans les archives de l'AMAE, leg. H-2524.

44. Voir le rapport de la Dirección General de Asuntos Políticos del Ministerio de Estado, daté du 13 septembre 1868, AMAE, leg. H-2524.

45. Gouvernement supérieur de Cuba au Sebastián de Mobellán, représentant officieux de l'Espagne au Mexique, 7 octobre 1868, AMAE, leg. H-2929. À propos de Santa Anna, voir W. Fowler, Santa Anna of Mexico, 2007.

46. Gouvernement supérieur de Cuba au ministre de l'Outre-mer, 28 septembre 1868, AMAE, leg. H-2929.

47. A. Sánchez, "Colonial Crisis and Spanish Diplomacy in the Caribbean During the Sexenio Revolucionario, 1868-1874 », Bulletin of Latin American Research, vol. 28, nº 3, 2009, p. 330.

\section{RÉSUMÉS}

Les réajustements de l'équilibre des pouvoirs dans les Caraïbes survenus après la chute de l'empire espagnol ont renforcé le conflit opposant l'Espagne, l'Angleterre, la France et les ÉtatsUnis pour le contrôle de cette zone stratégique. Entre 1821 et 1868 , la diplomatie espagnole adopte diverses stratégies défensives pour conserver ses colonies de Cuba et de Porto Rico, tout en résistant aux pressions britanniques qui visent à mettre fin à la traite des esclaves et aux besoins en main d'œuvre chez les élites esclavagistes cubaines. Tant l'expansionnisme des ÉtatsUnis sur le Mexique que le conflit anglo-américain en Amérique centrale ou les visées annexionnistes de Washington et de Madrid sur la République dominicaine contribuent alors à l'extrême volatilité des frontières régionales. Dans ce contexte délicat, les gouvernements espagnols successifs ont tenté d'obtenir de la France et de la Grande-Bretagne une garantie du maintien de la souveraineté espagnole sur Cuba et Porto Rico, tout en œuvrant à l'extension de l'influence espagnole sur le Mexique et la République dominicaine, dans le but de contrecarrer la pression croissante des États-Unis sur les colonies antillaises de l'Espagne.

Changes in the balance of power in the Caribbean after the disappearance of the Spanish colonial empire escalated the conflict between Spain, England, France and the United States for control of this strategic region. Between 1821 and 1868, Spanish diplomacy adopted various defensive strategies to keep Cuba and Puerto Rico and to meet British pressure to end the slave trade and the labor needs of the Cuban slave elite. U.S. expansionism on Mexico, the Anglo-American confrontation in Central and Washington and Madrid pressures on the Dominican Republic conferred highly volatile regional boundaries. In this complex scenario, successive Spanish governments sought a Franco-British guarantee the maintenance of Spanish sovereignty in Cuba and Puerto Rico and maneuvered to extend their influence over Mexico and the Dominican Republic to establish counterweights against the growing U.S. pressure on its Caribbean colonies. 
INDEX

Mots-clés : Espagne, politique extérieure, Caraïbes, XIXe siècle

Keywords : Spain, Foreign Policy, Caribbean nineteenth century

\section{AUTEURS}

\section{AGUSTÍN SÁNCHEZ ANDRÉS}

Instituto de Investigaciones Históricas, UMSNH 